\title{
Debt contracts with ex-ante and ex-post asymmetric information: an example. *
}

\author{
G. Carlier ${ }^{\dagger}$ L. Renou ${ }^{\ddagger}$
}

1st September 2004

\begin{abstract}
We consider a simple model of lending and borrowing combining two informational problems: adverse selection and costly state verification. Our analysis highlights the interaction between these two informational problems. We notably show that the higher the monitoring cost, the less discriminating the optimal menu of contracts is.
\end{abstract}

Keywords: debt contracts, diversity of opinions, screening, costly monitoring, pooling.

JEL classification: C7, D8, G3.

${ }^{*}$ We thank Anne Villamil for insightful comments.

†Université Paris IX-Dauphine, CEREMADE, Place du Maréchal De Lattre De Tassigny, 75775 Paris CEDEX 16, France, carlier@ceremade.dauphine.fr.

${ }^{\ddagger}$ School of Economics, Room G52, Napier Building, University of Adelaide, 5005 Australia, ludovic.renou@adelaide.edu.au. 


\section{Introduction}

In this paper, we consider a simple model of borrowing and lending in the presence of ex-ante and ex-post asymmetric information. We model the expost asymmetric information problem as a costly state verification problem, that is, a borrower freely observes the return of his risky investment while a lender has to pay a monitoring cost (see Townsend (1979)). As for the ex-ante asymmetric information, we suppose that a lender and a borrower have different and privately known opinions about the possible returns of the risky investment. Differences in opinions might be explained by (un-modelled) differences in private information or, more simply, differences in subjective beliefs. Moreover, following, among others, Boyd and Smith (1993), we suppose that a borrower offers an incentive-compatible menu of contracts to a lender; hence, our problem turns out to be a screening problem together with a costly monitoring problem. It is precisely the aim of the paper to shed light on the interaction between the screening and the costly monitoring problems for the structure of optimal contracts.

Since a general analysis of the contracting problem is extremely complex, if not out of reach, this paper thus adopts specific assumptions, which give rise to closed-form solutions. For instance, we restrict ourselves to a special class of contracts, so-called secured simple debt contracts, and suppose that the distribution of project return is uniform. As a consequence, we obtain that, at the optimum, the borrower offers at most two contracts $\underline{\delta}$ and $\bar{\delta}$. In turn, this extreme case of bunching enables us to focus on two types of optima: pooling and separating optima. We then analyze under which conditions pooling or separating solutions prevail.

We notably show that the more costly the monitoring, the less discriminating the optimal menu of contracts is. More precisely, there exists a threshold $\gamma^{*}$ such that if the monitoring cost is above $\gamma^{*}$, the borrower offers a unique contract while for lower monitoring costs, the borrower offers two contracts. In particular, absent away the costly state verification problem (i.e., a zero monitoring cost), the optimal menu of contracts always features two contracts. We also show that, for monitoring costs lower than $\gamma^{*}$, there exist opinions of the lender that should be offered the contract $\bar{\delta}$ in a world without adverse selection, and are offered the contract $\underline{\delta}$ in a world with adverse selection. However, for monitoring costs greater than $\gamma^{*}$, the borrower offers the same contract whether there is adverse selection or not. Thus, for high monitoring costs, the costly state verification effect dominates the adverse selection effect.

In a related paper, Boyd and Smith (1993) also consider a model with 
adverse selection and costly state verification problems, and prove the optimality of simple debt contracts. However, their model differs as they suppose that the borrower (the principal) is privately informed of both his ability (his type) to undertake a project and the realized return of the project. Hence, their problem is essentially a signalling problem together with a costly monitoring problem while our problem is a screening problem together with a costly monitoring problem.

In section 2, we present the model. Section 3 solves for the optimal menu of contracts and presents some comparative statics.

\section{The model}

We consider a two-period economy with a unique borrower and a unique lender. The lender is endowed with a single unit of a good that might be used for both investment and consumption while the borrower has no initial endowment. They are assumed to be risk-neutral and to care only about second period consumption. Moreover, the borrower has access to an investment project requiring exactly one unit of the investment good to be undertaken. The project returns $\omega$ in the second period with $\omega$ a realization of the random variable $\tilde{\omega}$. The opportunity cost is set to $r$.

Ex-post asymmetric information. The borrower freely observes the project return $\omega$ in the second period while the lender has to pay an utility cost of $\gamma$ to perfectly monitor the realized return $\omega$.

Ex-ante asymmetric information. The borrower and the lender have different, privately known, opinions (subjective beliefs) about the likelihood of exogenous factors, such as technology, consumers' taste, etc., which influence the project return. For instance, the lender might have (un-modelled) private information about the returns of similar projects undertaken in the past or might use his own model or method to assess the likelihood of these exogenous factors. Similar considerations apply to the borrower. For simplicity, we summarize these factors by a discrete random variable $\theta$, which takes values in $\left\{\theta_{1}, \ldots, \theta_{N}\right\}$ and is not contractible. Moreover, it is common knowledge that, for each realization $\theta_{n}$ of $\theta$, the probability density $g_{n}$ of the project return $\omega$ conditional on $\left[\theta=\theta_{n}\right]$ is uniform on $\left[\theta_{n}-\varepsilon, \theta_{n}+\varepsilon\right]$. We assume, furthermore, that $\theta_{1}<\theta_{2}<\ldots \theta_{n}<\ldots<\theta_{N}$ (means are ordered), that $\theta_{1} \geq \varepsilon$ (the return is almost surely non-negative) and $\theta_{N}-\theta_{1}<2 \varepsilon$ (the intersection of the supports is non-empty).

We also define $p_{n}$ (resp. $q_{n}$ ) as the probability of $\theta_{n}$ for the lender (resp. the borrower), and $p:=\left(p_{1}, \ldots, p_{N-1}\right)$ (resp. $\left.q\right)$ a point in $\Sigma_{N-1}$ the simplex 
of dimension $N-1$. It follows that the lender and the borrower have different opinions about the expected return of the project. The borrower believes that the expected return to undertake the risky project is $\sum_{n=1}^{N} q_{n} \theta_{n}$ while the lender expected return is $\sum_{n=1}^{N} p_{n} \theta_{n}$. We also assume that the borrower believes that the project expected return is greater than the opportunity cost. Finally, since opinions are private knowledge, we suppose that the borrower believes that the lender's opinion (type) is drawn from a non-degenerate probability measure with density $\rho$ with respect to the Lebesgue measure on $\Sigma_{N-1}$.

Contracts. Following, among others, Boyd and Smith (1993), we assume that the borrower offers a menu of contracts to the lender. Since our purpose is to shed light on the interplay between the problems of ex-ante and ex-post asymmetric information, we restrict ourselves to a simple class of contracts, so-called secured simple debt contracts (SDC), which gives rise to closed-form solutions.

Definition 1 A secured simple debt contract is a pair $(\bar{\omega}, C)$ with $C$ the collateral, $\min (\omega, \bar{\omega})$ the contingent repayment and $\{\omega: \omega<\bar{\omega}\}$ the monitoring states.

In a secured SDC, the borrower offers a non-contingent payment (or collateral) $C$ to the lender, a repayment of $\omega$ in monitoring states $\{\omega: \omega<\bar{\omega}\}$ and a fixed payment $\bar{\omega}$ in non-monitoring states. Note that if the lender is offered an actual return of $\omega<\bar{\omega}$, he commits to monitor the project and pays the monitoring cost. Several additional remarks are in order. First, observe that a secured simple debt contract is truth-telling, i.e., the borrower has no incentive to misreport the project return (see, for instance, Gale and Hellwig (1985)). Second, it is clear that in non-monitoring states, the borrower will repay a fixed amount to the lender. This observation holds true in any costly state verification model. We also assume that the lender seizes all the return realized in monitoring states. In a model without diversity of opinions, Gale and Hellwig (1985) and Williamson (1986) have shown that it is indeed optimal to seize all the return in non-monitoring states. However, this result might not hold in more general models (see Carlier and Renou (2005)). Third, the class of contracts we consider is richer than the standard class of SDC (see for instance Gale and Hellwig (1985), Krasa and Villamil (1992)) since the borrower can offer a secured part $C$ to the lender, while he is not allowed in the standard class of SDC. Finally, real-world counterparts of (secured) SDCs are (secured) corporate bonds, which are increasingly used by corporations. Indeed, the corporate bond market is large and liquid, which daily trading volume estimated at $\$ 23$ billion. Issuance for 2002 was 
an estimated $\$ 594$ billion ( $\$ 58$ in 1982), and the total market value in the US at the end of 2002 was approximately $\$ 4.1$ trillion (approximately, $\$ 0.5$ trillion in 1982). ${ }^{1}$ Moreover, note that most corporate bonds are debenture bonds i.e., fixed-interest security issued by corporations in return for loan. Debenture interest must be paid whether the corporation makes a profit or not, and in the event of non-payment, debenture holders can force liquidation. Finally, let us mention that secured corporate bonds are also frequently called collateral trust bonds.

For the sake of tractability, we impose that $\bar{\omega} \in[\underline{\delta}, \bar{\delta}]$ with $^{2}$

$$
[\underline{\delta}, \bar{\delta}] \subset \bigcap_{n}\left[\theta_{n}-\varepsilon, \theta_{n}+\varepsilon\right] .
$$

Thus, in our simple model, the borrower wishes to discriminate among the different types of a lender (screening) i.e., he does not want to remunerate too much a lender who would have lend him the funds at a lower cost. However, as will see later, the costly state verification problem renders more difficult the discrimination.

\section{The borrower's program}

The expected utility of a lender of type $p$, facing the contract $(\bar{\omega}, C)$ (with $\bar{\omega} \in[\underline{\delta}, \bar{\delta}])$ is

$$
U(p, \bar{\omega}, C)=\sum_{n=1}^{N} \frac{p_{n}}{2 \varepsilon}\left(\int_{\theta_{n}-\varepsilon}^{\bar{\omega}}(\omega-\gamma) d \omega+\int_{\bar{\omega}}^{\theta_{n}+\varepsilon} \bar{\omega} d \omega\right)+C .
$$

Denoting $x \cdot y$ the inner product of $(x, y) \in\left(\mathbb{R}^{N-1}\right)^{2}$, we can rewrite $U$ as

$$
U(p, \bar{\omega}, C)=(\alpha \cdot p) \bar{\omega}+\beta \cdot p+z(\bar{\omega})+C,
$$

where we have set for $n=1, \ldots, N-1$ :

$$
\begin{aligned}
\alpha_{n} & :=\frac{1}{2 \varepsilon}\left(\theta_{n}-\theta_{N}\right), \beta_{n}=P\left(\alpha_{n}\right), P(\alpha):=\alpha\left(\gamma+\varepsilon-\theta_{N}-\varepsilon \alpha\right), \\
z(\bar{\omega}) & =\frac{1}{2 \varepsilon}\left[-\frac{1}{2} \bar{\omega}^{2}+\bar{\omega}\left(\theta_{N}+\varepsilon-\gamma\right)-\frac{1}{2}\left(\theta_{N}-\varepsilon\right)^{2}+\gamma\left(\theta_{N}-\varepsilon\right)\right] .
\end{aligned}
$$

Note that the $\alpha_{n}$ 's are negative.

\footnotetext{
${ }^{1}$ Source: An investor's guide to Corporate Bonds, The Bond Market Association, www.bondmarket.com

${ }^{2}$ Note that $\bar{\omega} \in[\underline{\delta}, \bar{\delta}]$ implies that $\theta_{n}-\varepsilon \leq \bar{\omega} \leq \theta_{n}+\varepsilon$ for all $\theta_{n}$. In turn, this implies that, for any realization of $\theta$, the surplus is nonincreasing in the monitoring cost. For otherwise, we have the counter-intuitive result that the higher the monitoring cost, the higher the surplus.
} 


\subsection{Admissible contracts}

We define the set of admissible menus of contracts as the set of maps $p \mapsto$ $(\bar{\omega}(p), C(p))$ that satisfy $\bar{\omega}(p) \in[\underline{\delta}, \bar{\delta}]$ for all $p \in \Sigma_{N-1}$, are incentive-compatible (4) and individually rational (5), that is, for all types $\left(p, p^{\prime}\right)$ :

$$
\begin{gathered}
U(p, \bar{\omega}(p), C(p)) \geq U\left(p, \bar{\omega}\left(p^{\prime}\right), C\left(p^{\prime}\right)\right), \\
U(p, \bar{\omega}(p), C(p)) \geq r .
\end{gathered}
$$

Suppose that $(\bar{\omega}(),. C()$.$) is incentive-compatible, and, for every type p$, define

$$
V(p):=U(p, \bar{\omega}(p), C(p))=(\alpha \cdot p) \bar{\omega}(p)+\beta \cdot p+z(\bar{\omega}(p))+C(p) .
$$

the utility of a lender of type $p$ associated to the contract $(\bar{\omega}(p), C(p))$. Using (1) and (4), we obviously have

$$
V(p)=\sup \left\{(\alpha \cdot p) \bar{\omega}\left(p^{\prime}\right)+z\left(\bar{\omega}\left(p^{\prime}\right)\right)+C\left(p^{\prime}\right): p^{\prime} \in \Sigma_{N-1}\right\}+\beta \cdot p .
$$

Since a supremum of affine functions is convex, $V$ can be written as:

$$
V(p)=f(\alpha \cdot p)+\beta \cdot p,
$$

for some convex function $f:\left[\alpha_{1}, 0\right] \rightarrow \mathbb{R}$. Moreover, incentive-compatibility together with (8) and the Envelope Theorem yield that for a.e. $p \in \Sigma_{N-1}$ :

$$
\nabla V(p)=f^{\prime}(\alpha \cdot p) \alpha+\beta=\bar{\omega}(p) \alpha+\beta .
$$

Hence $f^{\prime}(\alpha \cdot p)=\bar{\omega}(p)$ and, thus, $\bar{\omega}(p)$ depends only on the reduced type $t:=\alpha \cdot p$. In words, the borrower discriminates different types of the lender in a single (unidimensional) dimension, the reduced type $t$, which is perfectly and positively correlated with the lender's expectation about the project return. Two types with the same expected return will be offered the same contract. Slightly abusing notations, we write $\bar{\omega}(t)=f^{\prime}(t)$ as a function of the unidimensional parameter $t$. Using $\bar{\omega}(t)=f^{\prime}(t),(6)$ and (8) also implies that the collateral only depends on $t$ through the formula ${ }^{3}$ :

$$
C(t)=f(t)-t f^{\prime}(t)-z\left(f^{\prime}(t)\right) .
$$

\footnotetext{
${ }^{3}$ From (10), it is worth noting that, due to incentive compatibility, we cannot easily impose an arbitrary value to $C$, say $C=r$. Indeed, in this case, on top of being convex, $f$ would have to solve the nonlinear differential equation $f(t)-t f^{\prime}(t)-z\left(f^{\prime}(t)\right)=r$. If this nonlinear differential equation has no solution, then the set of incentive-compatible contracts is simply empty, hence there is no optimal menu. Allowing for type-dependent collateral adds more degrees of freedom to the borrower's contract space such that we avoid the above problem. This is an important departure from the standard CSV literature, in which using collateral would eliminate monitoring.
} 
Conversely, it is standard to check that if $f$ is convex, $f^{\prime} \in[\underline{\delta}, \bar{\delta}], f^{\prime}=\bar{\omega}$ and (10) holds (again with $t:=\alpha \cdot p$ ), then the contract $(\bar{\omega}(),. C()$.$) is indeed$ incentive-compatible (see Rochet (1987)).

As for the individual rationality, observe that the constraint (5) is equivalent to $f(\alpha \cdot p)+\beta \cdot p \geq r$ for every type $p$.

Lemma 1 Let $f:\left[\alpha_{1}, 0\right] \rightarrow \mathbb{R}$ be a Lipschitz function such that $f^{\prime} \geq \underline{\delta}$, then

$$
\min _{p \in \Sigma_{N-1}} f(\alpha \cdot p)+\beta \cdot p \geq r
$$

if and only if $f\left(\alpha_{1}\right) \geq r-\beta_{1}$.

Proof. Let us write the previous condition as:

$$
f(x)+y \geq r, \text { for all }(x, y) \in \mathcal{C},
$$

where $\mathcal{C}:=\left\{(\alpha \cdot p, \beta \cdot p), p \in \Sigma_{N-1}\right\}$. It is direct to check that $\mathcal{C}$ is the convex hull of the points $(0,0)=(0, P(0))$ and $\left(\alpha_{n}, \beta_{n}\right)_{n=1, \cdots, N-1}=\left(\alpha_{n}, P\left(\alpha_{n}\right)\right)_{n=1, \cdots, N-1}$ where $P$ is the concave quadratic function defined by (2). Defining for all $x \in\left[\alpha_{1}, 0\right]$

$$
\Gamma(x):=\min \{y:(x, y) \in \mathcal{C}\},
$$

then condition (11) can be rewritten as:

$$
f(x)+\Gamma(x) \geq r \text { for all } x \in\left[\alpha_{1}, 0\right]
$$

We claim that $\Gamma$ is linear, more precisely

$$
\Gamma(x)=\frac{P\left(\alpha_{1}\right)}{\alpha_{1}} x \text { for all } x \in\left[\alpha_{1}, 0\right]
$$

First, since $(0,0)$ and $\left(\alpha_{1}, P\left(\alpha_{1}\right)\right)$ belong to $\mathcal{C}$, which is convex, $\left(x, \frac{P\left(\alpha_{1}\right)}{\alpha_{1}} x\right) \in \mathcal{C}$ for all $x \in\left[\alpha_{1}, 0\right]$ so that $\Gamma(x) \leq \frac{P\left(\alpha_{1}\right)}{\alpha_{1}} x$. Second, let us prove the converse inequality i.e. $y \geq \frac{P\left(\alpha_{1}\right)}{\alpha_{1}} x$ for all $(x, y) \in \mathcal{C}$. Since it is a linear inequality it is enough to check it at the vertices of $\mathcal{C}$, for $(x, y)=(0,0)$ or $(x, y)=\left(\alpha_{1}, \beta_{1}\right)$ there is nothing to check, for the vertices $\left(\alpha_{n}, P\left(\alpha_{n}\right)\right)$ the desired inequality follows from the concavity of $P$ and the fact that $\alpha_{n} \in\left[\alpha_{1}, 0\right]$.

Using (2) and since $\alpha_{1}<0$, we have:

$$
\frac{P\left(\alpha_{1}\right)}{\alpha_{1}}=\gamma+\varepsilon-\theta_{N}-\varepsilon \alpha_{1} \geq \gamma+\varepsilon-\theta_{N} .
$$

But since $f^{\prime} \geq \underline{\delta} \geq \theta_{N}-\varepsilon$, we obtain that $f+\Gamma$ is nondecreasing, hence achieves its minimum at $\alpha=\alpha_{1}$. It thus follows that the condition $f+\Gamma \geq r$ is equivalent to $f\left(\alpha_{1}\right)+\Gamma\left(\alpha_{1}\right) \geq r$, and since $\Gamma\left(\alpha_{1}\right)=P\left(\alpha_{1}\right)=\beta_{1}$ the proof is achieved. 


\subsection{Optimal contracts}

Within the class of admissible contracts, the borrower maximizes his expected profit, which is given by:

$$
\Pi=\int_{\Sigma_{N-1}}\left[\sum_{n=1}^{N} \frac{q_{n}}{2 \varepsilon} \int_{\bar{\omega}(p)}^{\theta_{n}+\varepsilon}(\omega-\bar{\omega}(p)) d \omega-C(p)\right] \rho(p) d p .
$$

Let us define the probability measure $\mu$ on $\left[\alpha_{1}, 0\right]$ as the image of $\rho(p) d p$ by the linear form $p \mapsto \alpha \cdot p$, that is, for every continuous function $\varphi$ on $\left[\alpha_{1}, 0\right]$ :

$$
\int_{\alpha_{1}}^{0} \varphi(t) d \mu(t)=\int_{\Sigma_{N-1}} \varphi(\alpha \cdot p) \rho(p) d p
$$

Using the reduced type $t, f^{\prime}=\bar{\omega}$ and (10), the borrower's profit becomes

$$
\Pi=\int_{\alpha_{1}}^{0}\left[\left(t+\frac{1}{2 \varepsilon}\left(\theta_{N}-\sum_{n=1}^{N} \theta_{n} q_{n}-\gamma\right)\right) f^{\prime}(t)-f(t)\right] d \mu(t)+k
$$

where $k$ is a constant. ${ }^{4}$ At this point, it is worth pointing out that $\Pi$ is linear with respect to $f$; once again this is very specific to the uniform assumption (quadratic terms in $f^{\prime}$ vanish). This linearity will of course dramatically simplify the structure of optimal contracts.

Let us assume, for simplicity, that $\mu$ has a density ${ }^{5} g_{\mu}$ and denote by $G_{\mu}$ the cumulative function of $\mu$. Integrating (14) by parts gives:

$\Pi=-f\left(\alpha_{1}\right)+\int_{\alpha_{1}}^{0}\left[G_{\mu}(t)-1+\left(t+\frac{1}{2 \varepsilon}\left(\theta_{N}-\sum_{n=1}^{N} \theta_{n} q_{n}-\gamma\right)\right) g_{\mu}(t)\right] f^{\prime}(t) d t+k$.

Optimal contracts obviously are such that the participation constraint is binding at the bottom : $f\left(\alpha_{1}\right)=r-\beta_{1}$ (use Lemma 1 ) and taking $u:=f^{\prime}$ as new unknown (hence $u=f^{\prime}=\bar{\omega}$ ), the borrower's program becomes:

$$
\max _{u \in K} L(u):=\int_{\alpha_{1}}^{0} h(t) u(t) d t
$$

\footnotetext{
4

$$
k:=\frac{1}{4 \varepsilon}\left(\sum_{n=1}^{N}\left(\theta_{n}+\varepsilon\right)^{2} q_{n}-\left(\theta_{N}-\varepsilon\right)\left(\theta_{N}-\varepsilon-2 \gamma\right)\right) .
$$

${ }^{5}$ It can be shown that $\mu$ is absolutely continuous with respect to Lebesgue measure, and that its density $g_{\mu}$ is continuous if $\rho$ is continuous. Moreover, it can be checked that this density vanishes at endpoints $g_{\mu}(0)=g_{\mu}\left(\alpha_{1}\right)=0$. Proofs of those facts are available upon request from the authors.
} 
with

$$
h(t):=G_{\mu}(t)-1+\left(t+\frac{1}{2 \varepsilon}\left(\theta_{N}-\sum_{n=1}^{N} \theta_{n} q_{n}-\gamma\right)\right) g_{\mu}(t)
$$

and:

$$
K:=\left\{u:\left[\alpha_{1}, 0\right] \rightarrow[\underline{\delta}, \bar{\delta}], u \text { is nondecreasing }\right\} .
$$

Since the borrower program (15) is a simple linear program, the maximum is achieved in at least one extreme point of $K$. Moreover Krein-Millman's Theorem (see [6]) and weak compactness of $K$ in $L^{p}$ imply that the set of solutions of (15) (which is a face of $K$ ) is the closed convex hull of the set of extreme points which solve (15). Hence, we focus on solutions in the set of extreme points of $K$. It is easy to check that those extreme points are the nondecreasing functions taking values in $\{\underline{\delta}, \bar{\delta}\}$ only. In other words, the set of extreme points of $K$ consists of the family of step functions $\left\{\underline{\delta} \mathbf{1}_{\left[\alpha_{1}, t\right)}+\bar{\delta} \mathbf{1}_{[t, 0]}, t \in\left[\alpha_{1}, 0\right]\right\}$. As a first consequence, the borrower's program (15) admits at least one solution which only takes values $\underline{\delta}$ and $\bar{\delta}$ (put differently, there always exists an optimal menu of contracts with at most two contracts.) More interestingly, restricting the maximization to extreme points of $K,(15)$ simply amounts to maximize over $\left[\alpha_{1}, 0\right]$, the function:

$$
F(t):=\underline{\delta} \int_{\alpha_{1}}^{t} h(s) d s+\bar{\delta} \int_{t}^{0} h(s) d s \text { for all } t \in\left[\alpha_{1}, 0\right],
$$

Denoting $A$ the argmax of $F$ over $\left[\alpha_{1}, 0\right]$, we get that the set of solutions of (15) is the closed convex hull (in the $L^{1}$ topology, say) of $\left\{\underline{\delta} \mathbf{1}_{\left[\alpha_{1}, t\right)}+\bar{\delta} \mathbf{1}_{[t, 0]}, t \in\right.$ $A\}$. If $A$ is not reduced to a singleton, say $\left(t, t^{\prime}\right) \in A^{2}$ with $t<t^{\prime}$, then both step functions $\underline{\delta} \mathbf{1}_{\left[\alpha_{1}, t\right)}+\bar{\delta} \mathbf{1}_{[t, 0]}$ and $\underline{\delta} \mathbf{1}_{\left[\alpha_{1}, t^{\prime}\right)}+\bar{\delta} \mathbf{1}_{\left[t^{\prime}, 0\right]}$ are solutions of (15), and any convex combination of those step functions is also optimal. Taking convex combinations amounts to adding an intermediate third value to the function. In the case where a menu with three or more contracts yields the same profit as a simpler menu, the borrower is more likely to offer the simplest one (remember we abstract from writing costs). For simplicity, we shall only discuss those simplest menus.

Finally, note that $F$ is differentiable and:

$$
F^{\prime}(t)=(\underline{\delta}-\bar{\delta})\left(G_{\mu}(t)-1+\left(t+\frac{1}{2 \varepsilon}\left(\theta_{N}-\sum_{n=1}^{N} \theta_{n} q_{n}-\gamma\right)\right) g_{\mu}(t)\right) .
$$

Hence, since $g_{\mu}(0)=g_{\mu}\left(\alpha_{1}\right)=0, G_{\mu}(0)=1$ and $G_{\mu}\left(\alpha_{1}\right)=0$ we have $F^{\prime}\left(\alpha_{1}\right)=(\bar{\delta}-\underline{\delta})>0$, and $F^{\prime}(0)=0$. This proves indeed that $\alpha_{1} \notin A$, i.e. 
the constant function $u \equiv \bar{\delta}$ is not a solution of (15). Note also that if the condition

$$
\theta_{N}-\sum_{n=1}^{N} \theta_{n} q_{n} \leq \gamma
$$

is satisfied, then $F$ is increasing, hence $A=\{0\}$ and the only optimal menu of contracts is a single contract such that $\bar{\omega} \equiv \underline{\delta}$ (pooling case).

\section{Theorem 1}

- The constant function $u \equiv \bar{\delta}$ is not a solution of (15),

- If (19) is satisfied, then (15) admits as unique solution the constant function $u \equiv \underline{\delta}$ (pooling case).

- If, in addition, $g_{\mu}$ is nonincreasing in a neighborhood of $0^{6}$, and (19) does not hold then $u \equiv \underline{\delta}$ is not a solution of (15), hence every solution of (15) takes at least two values (separating case).

Proof. Only the last statement has not been established yet. Assume:

$$
\theta_{N}-\sum_{n=1}^{N} \theta_{n} q_{n}>\gamma
$$

It is enough to prove that $F$ does not achieve its maximum at 0 . For $\alpha_{1}<$ $t<0$ compute

$$
\frac{F^{\prime}(t)}{(\bar{\delta}-\underline{\delta}) g_{\mu}(t)}=\left(\frac{1-G_{\mu}(t)}{g_{\mu}(t)}-\left(t+\frac{1}{2 \varepsilon}\left(\theta_{N}-\sum_{n=1}^{N} \theta_{n} q_{n}-\gamma\right)\right)\right)
$$

Since

$$
0 \leq \frac{1-G_{\mu}(t)}{g_{\mu}(t)}=\frac{\int_{t}^{0} g_{\mu}}{g_{\mu}(t)}
$$

our assumption implies that for $t$ close to 0

$$
0 \leq \frac{1-G_{\mu}(t)}{g_{\mu}(t)} \leq-t \text { hence } \lim _{t \rightarrow 0^{-}} \frac{F^{\prime}(t)}{(\bar{\delta}-\underline{\delta}) g_{\mu}(t)}<0 .
$$

This implies that $F^{\prime}(t) / g_{\mu}(t)<0$ for $t$ close to 0 so that $0 \notin A$. This ends the proof.

\footnotetext{
${ }^{6}$ It can be checked that these assumptions are satisfied if $\rho$ is smooth and strictly positive: proofs are available from the authors upon request.
} 


\subsection{Comparative statics}

Theorem 1 exhibits two types of solutions: solutions of the pooling type and solutions of the separating type. In a pooling solution, the borrower offers a unique contract to the lender while, in a separating solution, the borrower offers two contracts. The monitoring cost $\gamma$ as well as the relative optimism/pessimism $\theta_{N}-\sum_{n=1}^{N} q_{n} \theta_{n}$ of the borrower determine the type of solution (see condition (19)). For high monitoring costs and/or a relatively pessimistic borrower, pooling solutions prevail, and the borrower offers the contract $\underline{\delta}$; whereas for low monitoring costs and/or a relatively optimistic borrower, separating solutions prevail, and the borrower offers two contracts $\underline{\delta}$ for reduced types $t$ below $t^{*}$ with $t^{*} \in A$ and $\bar{\delta}$, otherwise. It is important to note that this extreme case of bunching is rather specific to the assumptions of our model. We think that a more general model (if such a more general model can be solved) is likely to feature less bunching.

Note that if $\gamma=0$ and $q \neq 0$, condition (19) is fulfilled. In words, if there is no costly state verification problem, the borrower optimally offers two contracts. More generally, for a fixed $q$, there exists a $\gamma^{*}>0$ such that for all $\gamma>\gamma^{*}$, the optimal menu of contracts is the singleton menu $\underline{\delta}$ while for all $\gamma<\gamma^{*}$, the borrower offers two contracts $\underline{\delta}$ and $\bar{\delta}$.

Before going further, consider the optimal contracts when the borrower knows the type of the lender i.e., absent away the adverse selection problem. Optimal contracts maximize the borrower expected profit (13) subject to the participation constraint (5). It is easy to show ${ }^{7}$ that the optimal contract is $\underline{\delta}$ if $t<\tau$ and $\bar{\delta}$, otherwise, with

$$
\tau=-\frac{1}{2 \varepsilon}\left(\theta_{N}-\sum_{n=1}^{N} \theta_{n} q_{n}-\gamma\right) .
$$

Observe that if $\tau \geq 0$, the borrower offers the contract $\underline{\delta}$ to the lender whatever his type. The condition $\tau \geq 0$ is equivalent to condition (19). In particular, if (19) holds, the borrower offers a unique contract regardless of the type of the lender while if (19) does not holds, the contract offered depends on the type of the lender. From now on, we suppose that (19) does not hold.

A maximum $t^{*}$ of $F$ is a solution of

$$
t^{*}-\frac{1-G_{\mu}\left(t^{*}\right)}{g_{\mu}\left(t^{*}\right)}=\tau .
$$

\footnotetext{
${ }^{7} \mathrm{~A}$ proof is available upon request
} 
The left-hand side of (20) could be interpreted as the lender's type that takes into account the incentive compatibility constraints (equivalently, the informational rents), that is the virtual type. Hence, condition (20) says that the virtual type for which the borrower is indifferent between offering the low or high contract is precisely $\tau$, the type for which the borrower is indifferent between offering the low or high contract, absent away the problem of adverse selection. Note also that we necessarily have $t^{*} \geq \tau$. Thus, there possibly exists a range of types, which would be offered the contract $\bar{\delta}$ in a world without adverse selection but are offered $\underline{\delta}$ in a world with adverse selection.

Finally, observe that $F$ is strictly supermodular in the reduced type $t$ and the monitoring cost $\gamma$ (use formula (18)), hence $A$, the set of maxima of $F$, is increasing in $\gamma$.

Our paper is thus a first step towards a systematic analysis of the impact of ex-ante and ex-post asymmetric information for the structure of optimal contracts. As it is already apparent from our analysis, this impact is not trivial and interested. It certainly deserves further research.

\section{References}

[1] J. Boyd and B.D. Smith, The Equilibrium Allocation of Investment Capital in the Presence of Adverse Selection and Costly State Verification, Economic Theory, 3(3), 1993, 427-51.

[2] G. Carlier and L. Renou, A Costly State Verification Model with Diversity of Opinions, Economic Theory, 25(2), 2005, 497-504.

[3] D. Gale and M. Hellwig, Incentive-Compatible Debt Contracts: The OnePeriod Problem, Review of Economics Studies, 52(4), 1985, 647-663.

[4] S. Krasa and A. Villamil, Monitoring the Monitor: An Incentive Structure for a Financial Intermediary, Journal of Economic Theory, 57(1), 1992, $197-221$.

[5] J.-C. Rochet, A Necessary and Sufficient Condition for Rationalizability in a Quasi-Linear Context, Journal of Mathematical Economics, 16(2), 1987, 191-200.

[6] H.L. Royden, Real Analysis, Third Edition, Prentice Hall (1988).

[7] R. Townsend, Optimal Contracts and Competitive Markets with Costly State Verification, Journal of Economic Theory, 21(2), 1979, 265-93. 